\title{
Análisis comparativo de las guías de la ESPEN y la Academia de Nutrición y Dietética Americana sobre cuidado nutricional del paciente con cáncer publicadas en 2017
}

\author{
Comparative analysis of the ESPEN guidelines on nutrition in cancer patients \\ with the American Academy of Nutrition and Dietetics Oncology Evidence- \\ Based Nutrition Practice Guideline for Adults published in 2017
}

\author{
Mónica Bejarano Rosales' ${ }^{1}$ Karolina Álvarez Altamirano ', Vanessa Fuchs-Tarlovsky ${ }^{1 *}$ \\ Recibido: 5 de diciembre de 2018. Aceptado para publicación: 25 de enero de 2019 \\ Publicado en línea, febrero 14 de 2019 \\ https://doi.org/10.35454/rncm.v2n1.056
}

\section{Resumen}

Introducción: en los últimos años se han desarrollado guías clínicas y recomendaciones prácticas elaboradas de manera consensual que promueven y sensibilizan sobre la importancia del cuidado nutricional en los pacientes con cáncer.

Objetivo: comparar las guías de la Sociedad Europea de Nutrición Clínica y Metabolismo (European Society for Clinical Nutrition and Metabolism, ESPEN) y de la Academia de Nutrición y Dietética Americana (Academia) publicadas en 2017.

Metodología: se realizó un análisis cualitativo y comparativo de las guías ESPEN y de la Academia. Se presenta una síntesis de los principales resultados.

Resultados: se encontró que a pesar de que las guías comparten un objetivo común, se diferencian en el objetivo y la población objeto. Mientras las guías de la Academia tienen como objetivo actualizar y dar continuidad a las guías publicadas en años anteriores, centradas en el abordaje integral de la nutrición oncológica por parte del nutricionista, las guías de ESPEN tienen como objetivo traducir la evidencia actual y la opinión de expertos en recomendaciones dirigidas a equipos multidisciplinarios responsables de la identificación, prevención y tratamiento de la desnutrición del paciente oncológico.

Conclusión: se concluye que ambas guías proporcionan declaraciones sobre qué hacer en la práctica clínica, y orientar a las personas para que puedan auditar, comparar y mejorar potencialmente su desempeño profesional. Las guías, aunque presentan diferencias en ciertos temas, son una herramienta útil que orienta la práctica del cuidado nutricional en oncología.

Palabras clave: guías nutricionales, oncología, ESPEN, Academia, cuidado nutricional.

*Correspondencia: Vanessa Fuchs-Tarlovsky vanessafuchs@hotmail.com

\section{Summary}

In the last years, different societies published clinical guidelines to provide evidence into practical recommendations in a consensual manner that promote and raise awareness of the importance of nutritional care in cancer patients.

We compared the European Society for Clinical Nutrition and Metabolism (ESPEN) with those developed by the American Academy of Nutrition and Dietetics (Academy) published in 2017, and we found that both guides share a common objective, to establish practical recommendations based on evidence for the nutritional care in oncology patients. However, two main aspects differentiate them: the target audience and the thematic scope.

The aim of Academy guidelines to update the guides published in previous years and focus on addressing the integral practice of oncological nutrition. The ESPEN guidelines aim to translate the current evidence and the opinion of experts into recommendations for professionals of multidisciplinary teams responsible for the identification, prevention and treatment of malnutrition.

We concluded that both the ESPEN Guidelines and the Academy guides provide statements on what to do in clinical practice and which people can potentially audit compare and improve their practices. The guides, although they present differences in certain topics, are a useful tool that guides the practice of nutritional care in oncology.

Keywords: Nutritional guides; Oncology; ESPEN; Academy; Nutritional care. 


\section{INTRODUCCIÓN}

El cáncer es una de las primeras causas de muerte en el mundo. En el año 2012 se le atribuyeron 8,2 millones de muertes, de las cuales $70 \%$ se produjeron en África, Asia, América Central y Sudamérica. Según la Organización Mundial de la Salud (OMS) se prevé que el número de casos de cáncer aumente de los 14,1 millones registrados en 2012 a 21,6 millones en $2030^{(1)}$.

Los pacientes con cáncer tienen un riesgo particularmente alto de desnutrición porque tanto la enfermedad como sus tratamientos tienen implicaciones en su estado nutricional. Se estima que la muerte de $10 \%$ a $20 \%$ de los pacientes con cáncer se puede atribuir a la desnutrición más que a la malignidad en sí misma ${ }^{(2,3)}$. El impacto de la alteración del estado nutricional, en especial del peso y de la pérdida de la masa muscular, es importante sobre el pronóstico, la evolución, la calidad de vida y la tolerancia al tratamiento ${ }^{(4,5)}$. En el estudio de Martin et al, realizado en 8160 pacientes europeos y canadienses con estadios avanzados de cáncer, se muestra que un Índice de Masa Corporal (IMC) bajo $(<20$ $\mathrm{kg} / \mathrm{m}^{2}$ ) y el porcentaje de pérdida de peso predijeron de manera independiente la supervivencia general de estos pacientes ${ }^{(6)}$. Los pacientes con IMC $\geq 25,0 \mathrm{~kg} / \mathrm{m}^{2}$ y con un peso estable tuvieron la supervivencia más larga $(20,9 \text { meses, IC } 95 \%, 17,9 \text { a 23,9 meses })^{(6)}$.

A pesar de que la nutrición es considerada un aspecto importante en la atención integral del paciente con cáncer, estudios recientes en hospitales europeos encontraron que solo entre $30 \%$ y $60 \%$ de los pacientes con cáncer que estaban en riesgo de desnutrición recibieron apoyo nutricional: suplementos orales, nutrición parenteral y nutrición enteral ${ }^{(7)}$. En otro estudio europeo los médicos clasificaron en forma errónea, en $40 \%$ de los casos, la severidad de la desnutrición relacionada con el cáncer. Como resultado, muchos pacientes con desnutrición severa no recibieron las intervenciones nutricionales. Incluso cuando los médicos reconocieron la desnutrición relacionada con el cáncer, los pacientes y sus familiares a menudo subestimaron su presencia ${ }^{(8)}$.

Por lo tanto, aunque la oncología es una de las áreas de la medicina donde los avances terapéuticos recientes han logrado mejorar los resultados para los pacientes, la alta prevalencia de desnutrición que acompaña esta patología puede limitar su respuesta, en especial, si no se realiza un adecuado cuidado nutricional. Esto justifica la necesidad de un abordaje nutricional basado en estándares científicos ${ }^{(9,10)}$. En los últimos años, se han desarrollado guías clínicas que buscan traducir la evidencia científica en recomendaciones prácticas de manera consensual con el fin de promover y sensibilizar sobre la importancia de un adecuado cuidado nutricional. En las guías se presentan recomendaciones generales y un pequeño número de recomendaciones específicas para situaciones comunes ${ }^{(11-14)}$. Con el propósito de emitir guías para la práctica clínica más amplias y profundas, durante el año 2017, la Sociedad Europea de Nutrición Clínica y Metabolismo (ESPEN, por sus siglas en inglés) y la Academia de Nutrición y Dietética Americana (Academia) publicaron las guías prácticas sobre la nutrición en el paciente con cáncer ${ }^{(10,15)}$. Aunque el tratamiento nutricional de estos pacientes es la temática común de estas guías, se presentan diferencias que merece la pena analizar.

En consecuencia, el objetivo de este trabajo es hacer una comparación entre las recomendaciones de la guía de la ESPEN y de la Academia, publicadas en 2017, que permita analizar sus diferencias y similitudes y así poner en perspectiva el interés que tienen para la práctica clínica.

\section{METODOLOGÍA}

Se realizó un análisis cualitativo de las guías de la ESPEN, "ESPEN guidelines on nutrition in cancer patients" publicadas por Arends et al. en 2017 y de las guías de la Academia "Oncology Evidence-Based Nutrition Practice Guideline for Adults”, publicadas por Thompson et al. en el mismo año ${ }^{(10,15)}$. En este artículo se presenta una síntesis de los principales resultados.

\section{RESULTADOS}

\section{Comparación de la metodología utilizada en las guías}

La metodología utilizada en las guías de la ESPEN y de la Academia fue distinta. Las guías de la ESPEN cumplen con los ocho elementos estándar para el desarrollo de guías de la Academia Nacional de Medicina de Norteamérica ${ }^{(16)}$. El primer paso fue realizar una búsqueda bibliográfica de meta-análisis, revisiones sistemáticas y estudios comparativos basados en preguntas clínicas según el formato Populations of interest, interventions, comparisons, outcomes (PICO, por sus siglas en inglés). Los parámetros de las preguntas clínicas en este formato se muestran en la Tabla 1 . En un segundo paso, la evidencia fue evaluada y fusionada para desarrollar recomendaciones clínicas usando el método Grading of Recommendations Assessment, Development and Evaluation (GRADE, por sus siglas en inglés). Este 
Tabla 1. Características generales de las guías

\begin{tabular}{|c|c|}
\hline \multicolumn{2}{|c|}{ Definición de las estrategias de análisis y los parámetros de las preguntas clínicas de las guías de la ESPEN y de la Academia } \\
\hline ESPEN & Academia \\
\hline $\begin{array}{c}\text { Definición de los parámetros de las preguntas clínicas en } \\
\text { formato PICO }\end{array}$ & $\begin{array}{l}\text { Estrategia de la definición de preguntas clínicas de las guías } \\
\text { de la Academia }\end{array}$ \\
\hline $\begin{array}{l}\text { Población: mayores de } 18 \text { años. } \\
\text { Tipo de cáncer: hematológica, leucemia aguda, trasplante de } \\
\text { médula ósea. Sólidos: pulmonares, gastrointestinales, cabeza y } \\
\text { cuello y otros. } \\
\text { Condición: cuidado paliativo, curativo, sobreviviente y paciente } \\
\text { en fase terminal; capacidad funcional. } \\
\text { Tratamiento del cáncer: quimioterapia o radioterapia (causa de } \\
\text { náusea/anorexia); radioterapia en cabeza y cuello; radioterapia } \\
\text { en gastrointestinal; cirugía. } \\
\text { Estado nutricional: desnutrido, no desnutridos, anorexia. }\end{array}$ & $\begin{array}{l}\text { Revisión sistemática de la Biblioteca de Análisis de } \\
\text { Evidencia de la Academia de Nutrición y Dietética (EAL): } \\
\text { - Pacientes mayores de } 18 \text { años en cuidado ambulatorio, } \\
\text { cuidado agudo. Se excluye cuidado crítico, pacientes } \\
\text { cuidado paliativo, sobreviviente, paciente en fase terminal; } \\
\text { capacidad funcional. } \\
\text { - Pacientes con cáncer con y sin tratamiento. } \\
\text { - Antropometría: peso, masa magra, cambios. } \\
\text { - Terapia médica nutricional. Intervención dietética por RDN. } \\
\text { Con tratamiento quimio o radioterapia. } \\
\text { - Herramientas de tamizaje nutricional. } \\
\text { - Evaluación del estado nutricional. } \\
\text { - Nutrición terapéutica en pacientes con quimioterapia y } \\
\text { radioterapia. } \\
\text { - Suplementos orales con con aceite de pescado. }\end{array}$ \\
\hline $\begin{array}{l}\text { Intervenciones: soporte psicosocial, tamizaje, nutrición enteral, } \\
\text { nutrición parenteral, aumento de la ingesta calórica, aumento } \\
\text { de la ingesta proteica, glutamina, inmunonutrición, ácidos } \\
\text { grasos poliinsaturados omega-3, suplementos nutricionales. }\end{array}$ & $\begin{array}{l}\text { Análisis basado en revisiones sistemáticas realizadas por } \\
\text { organizaciones externas: } \\
\text { - Glutamina y mucositis oral en pacientes con tumores y } \\
\text { enfermedad hematológica. } \\
\text { - Dieta en neutropenia. }\end{array}$ \\
\hline $\begin{array}{l}\text { Desenlaces primarios: sobrevida general. } \\
\text { Secundarios: en orden de importancia: cambios en el peso, } \\
\text { peso corporal, índice de masa corporal, otros, sobrevida sin } \\
\text { enfermedad, calidad de vida, estado funcional, cumplimiento } \\
\text { con la terapia, complicaciones/estancia hospitalaria. }\end{array}$ & $\begin{array}{l}\text { Recomendaciones basadas en consensos: } \\
\text { - Tamizaje. } \\
\text { - Valoración según los estadios de cáncer (pre-caquexia, } \\
\text { caquexia). } \\
\text { - Diagnóstico de desnutrición. } \\
\text { - Intervención nutricional. }\end{array}$ \\
\hline \multicolumn{2}{|c|}{ Definición de desnutrición y caquexia del paciente adulto con cáncer } \\
\hline $\begin{array}{l}\text { La desnutrición es sinónimo de malnutrición. La caquexia es } \\
\text { considerada, según el consenso de ESPEN sobre terminología, } \\
\text { como un tipo de desnutrición crónica asociada a la enfermedad } \\
\text { con inflamación }{ }^{(24)} \text {. } \\
\text { Criterios diagnósticos: } \\
\text { Paso 1: Tamizaje nutricional, (NRS-2002, MUST, MNA, etc.) } \\
\text { Paso 2: Diagnóstico de desnutrición } \\
\qquad I M C<18,5 \mathrm{~kg} / \mathrm{m}^{2} \\
\quad 0, \\
\text { Pérdida de peso }>10 \% \text { en tiempo indefinido; }>5 \% \text { en los } \\
\text { últimos } 3 \text { meses, combinado con: } \\
\quad \text { IMC }<20 \text { ( }<70 \text { años) } /<22(>70 \text { años) } \\
\quad 0 \\
\text { Índice de masa libre de grasa: }<15 \text { y } 17 \mathrm{~kg} / \mathrm{m}^{2} \text { en } \\
\text { mujeres y hombres respectivamente. }\end{array}$ & $\begin{array}{l}\text { La desnutrición es un estado nutricional en el que la } \\
\text { deficiencia o exceso (o desequilibrio) de energía, proteína y } \\
\text { otros nutrientes causa efectos adversos medibles en la forma, } \\
\text { tamaño y composición de los tejidos o el cuerpo, su función } \\
\text { y los desenlaces clínicos. Los pacientes pueden además de la } \\
\text { desnutrición tener caquexia(15). } \\
\text { Criterios diagnósticos: } \\
\text { - La presencia de dos o más de los siguientes: } \\
\text { - Insuficiente consumo de energía. } \\
\text { - Pérdida de peso involuntaria. } \\
\text { - Pérdida de grasa subcutánea. } \\
\text { - Pérdida de masa muscular. } \\
\text { - Acumulación de líquido localizado o generalizado (que } \\
\text { puede enmascarar la pérdida de peso). } \\
\text { - Fuerza de agarre reducida. }\end{array}$ \\
\hline
\end{tabular}

enfoque ampliamente utilizado por instituciones como la OMS y el Cochrane Collaboration, permite calificar la calidad de la evidencia y la fuerza de las recomendaciones y es considerada como exhaustiva y transparente ${ }^{(17)}$.
La Academia aplicó una metodología que consta de cinco pasos descrita por Handu et al ${ }^{(18)}$. Los pasos son: formulación de la pregunta para el análisis de la evidencia; clasificación y agrupamiento de la evidencia (reco- 
pilación de datos); evaluación crítica de cada artículo (riesgo de sesgo); resumen de la evidencia; redacción y calificación de la conclusión. La búsqueda de evidencia se realizó en PubMed y Cumulative Index to Nursing y Allied Health Literature databases desde 1993 a 2011, y hasta 2013 para el tema de aceite de pescado. Para la revisión sistemática aplicaron la metodología de la Biblioteca de Análisis de Evidencia de la Academia de Nutrición y Dietética Americana (EAL, por sus siglas en inglés) ${ }^{(19,20)}$. El grupo de trabajo utilizó el consenso de expertos para expresar las declaraciones de recomendación condicional (define en forma clara una situación específica) o imperativa (ampliamente aplicable a una población objetivo, con restricciones sobre su pertinencia). Las recomendaciones se categorizaron de la siguiente manera: fuertes, razonables, de consenso o insuficientes (strong, fair, weak, consensus, or insufficient) según las rúbricas de calificación estandarizadas desarrolladas por la Academia.

La Academia utilizó tres estrategias para la definición de 16 preguntas clínicas: la revisión sistemática de la EAL, el análisis basado en revisiones sistemáticas realizadas por organizaciones externas y las recomendaciones basadas en publicaciones de consenso (Tabla 1).

\section{Objetivo y alcance de las guías}

Las dos guías comparten un objetivo común: establecer recomendaciones prácticas basadas en la evidencia para la atención nutricional del paciente oncológico. Sin embargo, las diferencian dos aspectos principales: el público al cual están destinadas y el ámbito temático.

Las guías de la ESPEN tienen como objetivo "traducir la evidencia actual y la opinión de expertos en recomendaciones dirigidas a equipos multidisciplinarios responsables de la identificación, prevención y tratamiento de los elementos reversibles de la desnutrición en pacientes con cáncer" ${ }^{\prime \prime 10}$. Además, tiene como objetivos específicos mejorar la detección temprana y el tratamiento de la desnutrición, y los trastornos metabólicos en pacientes con cáncer y los sobrevivientes; proporcionar orientación a los profesionales de la salud y a los pacientes sobre el manejo más apropiado y efectivo de los problemas nutricionales y metabólicos en pacientes con cáncer, y, con esto, reducir la incidencia y el impacto de la desnutrición y los trastornos metabólicos en estos pacientes. Para ello, la guía responde a preguntas clínicas precisas utilizando el formato PICO (Tabla 1).

Las guías de la Academia de Nutrición y Dietética Americana tienen como objetivo actualizar y dar conti- nuidad a las guías publicadas en 2007 y $2013^{(19,21)}$ y se centran en abordar la práctica integral de la nutrición oncológica para la atención de pacientes adultos con cáncer. Las estrategias del análisis y de la definición de las preguntas clínicas de las Guías de la Academia se muestran en la Tabla 1.

\section{Población objetivo}

Mientras que las guías de ESPEN están destinadas a los administradores de institutos oncológicos, los oncólogos clínicos, y profesionales de los equipos multidisciplinarios (médicos de familia, farmacéuticos, enfermeras, dietistas, nutricionistas y terapeutas físicos), las guías de la Academia están dirigidas a los nutricionistas-dietistas registrados (RDN, por sus siglas en inglés) $)^{(22)}$.

\section{Alcance y ámbito temático}

El alcance y ámbito de las guías no es el mismo, es decir, difieren en el campo o área temática que abarcan. Las guías de la ESPEN presentan recomendaciones de práctica clínica basadas en el enfoque multimodal del tratamiento nutricional del cáncer, es decir, también proporciona recomendaciones específicas sobre el cuidado nutricional que busca aumentar la ingesta nutricional, disminuir la inflamación y el estrés hipermetabólico, y aumentar la actividad física (Figura 1). Además, las guías de la ESPEN, no se limitan al tratamiento nutricional del paciente que recibe terapia anticancerosa, sino que aborda las distintas condiciones que se presentan en las fases del paciente con cáncer: cuidado paliativo, curativo, sobreviviente y estado terminal.

El grupo de trabajo de oncología de la Academia eligió enfocarse principalmente en cuatro áreas de nutrición oncológica en adultos donde había un grupo adecuado de evidencia relacionada con la nutrición: 1 . validez del tamizaje y evaluación del estado nutricional y sus herramientas, 2. la asociación entre el estado nutricional, la morbilidad y la mortalidad, 3. el efecto de la terapia médica nutricional en pacientes tratados con quimioterapia (CT) y radioterapia (RT), y 4. la caquexia en el paciente con cáncer y el efecto de suplementos dietéticos y alimentos con propósitos médicos especiales (APME) que contienen aceite de pescado (específicamente ácido eicosapentaenoico, EPA), en el peso corporal y masa corporal magra. Es de notar que la academia también concibe, a la terapia médica nutricional en el paciente oncológico adulto que recibe 

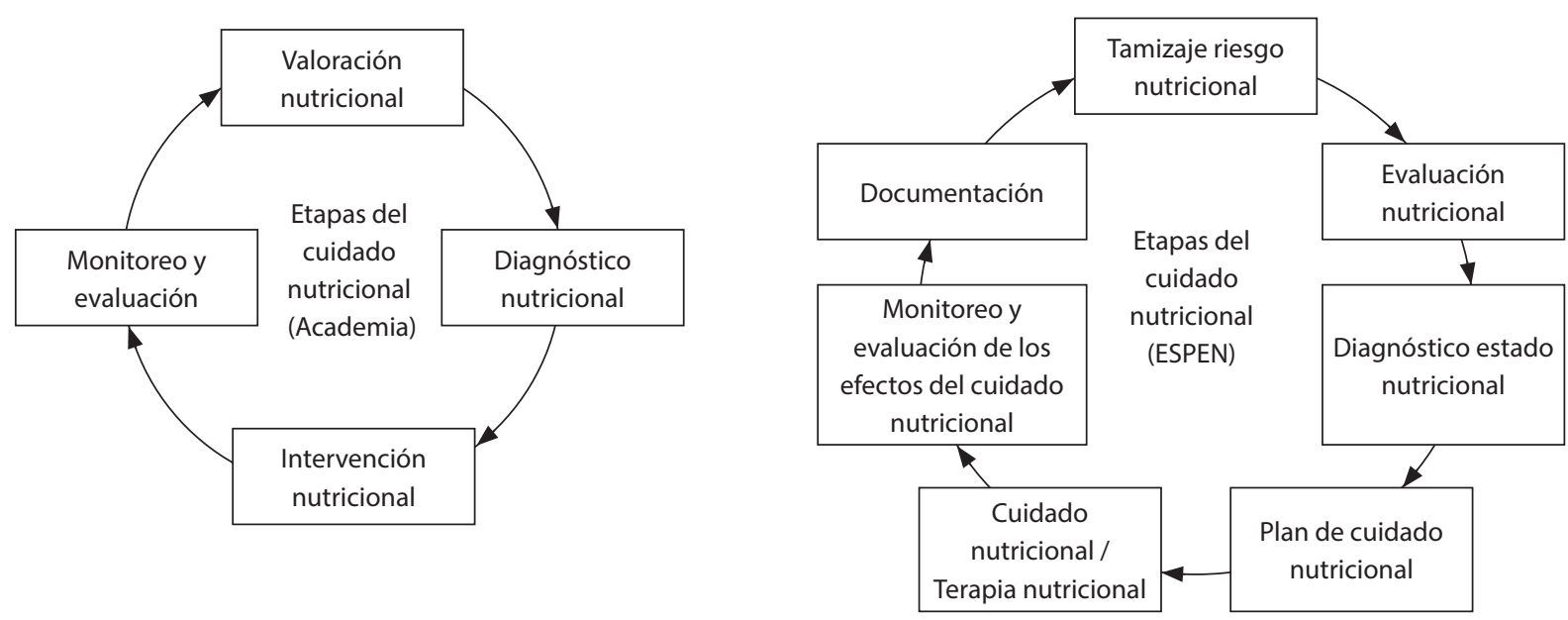

Público objetivo: nutricionistas

Alcance:

Tratamiento nutricional del paciente que recibe terapia

anticancerosa

- Validez del tamizaje y evaluación del estado nutricional y sus herramientas.

- Asociación entre estado nutricional, la morbilidad y mortalidad. - Efecto de la terapia médica nutricional en pacientes tratados con quimioterapia y radioterapia.

- La caquexia en el paciente con cáncer y el efecto de suplementos dietéticos que contiene grasas de pescado (EPA) en el peso.

Público objetivo: equipos interdisciplinarios Alcance:

Enfoque multimodal: (objetivos terapéuticos)

- Cuidado nutricional.

- Inflamación e hipermetabolismo.

- Actividad física.

Etapas del paciente oncológico:

- Cuidado paliativo.

- Etapa curativa (radio y quimioterapia).

- El paciente sobreviviente.

Figura 1. Etapas del proceso de cuidado nutricional según la Academia y ESPEN.

tratamiento con quimio y radioterapia, desde el enfoque multimodal. Sin embargo, estas guías no dan recomendaciones sobre los aspectos farmacológicos ni la actividad física del enfoque multimodal.

\section{El proceso de cuidado nutricional según la ESPEN y la Academia}

Las recomendaciones que proporcionan las dos guías se enmarcan en dos concepciones distintas del proceso de cuidado nutricional. El proceso de atención nutricional de la Academia fue publicado como parte del Modelo de Cuidados Nutricionales y está destinado a la práctica clínica del $\mathrm{RDN}^{(23)}$. El proceso proporciona un marco para que el RDN individualice la atención, teniendo en cuenta las necesidades del paciente y utilizando la mejor evidencia disponible para tomar decisiones. El proceso de cuidado nutricional de la ESPEN es también una secuencia sistemática que involucra distintos pasos interrelacionados. A diferencia de la Academia, en este proceso está involucrado todo el personal de salud que hace parte del cuidado nutricional e incluye otras etapas como el tamizaje y la documentación ${ }^{(24)}$ (Figura 1).

\section{La definición de desnutrición según la ESPEN y la Academia}

Las guías de la ESPEN utilizaron el estado nutricional como uno de los parámetros para definir las preguntas clínicas. Para este fin, se tuvieron en cuenta las siguientes posibilidades: pacientes desnutridos, no desnutridos y anorexia. La ESPEN a diferencia de la Academia, no utiliza la nueva terminología de sarcopenia, pre caquexia y caquexia refractaria; además, considera que estos términos todavía están en el nivel de propuesta $y$ no pueden presentarse en este momento como operativos. Por lo tanto, evita usar cualquiera de estos, a 
menos que se indique en forma explícita, y abordan en la guía, por separado, los componentes fisiopatológicos y clínicos de la desnutrición, incluida la inflamación sistémica, la anorexia, la ingesta de energía, la pérdida de la masa muscular / grasa y la actividad física reducida. La definición de malnutrición para la ESPEN y la Academia se muestran en la Tabla 1.

Se observa que mientras para la Academia la desnutrición y la caquexia son dos entidades distintas, entendiendo la caquexia como un estado de desgaste más avanzado, para la ESPEN la desnutrición es sinónimo de malnutrición y la caquexia (según su consenso sobre terminología) como un tipo de desnutrición crónica asociada a la enfermedad con inflamación. Estas diferencias son el reflejo de la discusión actual en la literatura científica sobre la necesidad de una definición homogénea de las distintas alteraciones del estado nutricional ${ }^{(24)}$.

\section{RECOMENDACIONES SOBRE TAMIZAJE, VALORACIÓN NUTRICIONAL, MONITOREO Y AUDITORIA EN PACIENTES ONCOLÓGICOS ADULTOS}

Para las dos guías, la necesidad de realizar tamizaje y evaluación del estado nutricional es un aspecto clave en la atención nutricional del paciente con cáncer. Además, las dos resaltan la importancia de iniciar un tratamiento nutricional temprano para evitar la pérdida de peso y de masa muscular. Sin embargo, recomiendan herramientas distintas. Estas diferencias son el reflejo de la ausencia de consenso internacional sobre la validación y el uso de las herramientas de tamizaje (Tabla 2). El tamizaje conduce a un diagnóstico del estado nutricional el cual, para las dos guías, debe tener un componente de antropometría, evaluación del estado físico funcional, evaluación de la ingesta y parámetros bioquímicos. La Academia además, tiene en cuenta la historia social incluyendo factores psicológicos y socioeconómicos.

Las dos guías concuerdan en que el monitoreo y la auditoria son parte importante del proceso de cuidado nutricional pero aún no existen parámetros validados. Ambas proponen, por consenso, una aproximación de los criterios de estas etapas.

\section{Recomendaciones de requerimientos energéticos, y de substratos}

La guía de la ESPEN proporciona recomendaciones específicas sobre el gasto energético total, los requerimientos de proteína, substratos energéticos y vitaminas, mientras que la Academia abarca solo recomendaciones sobre vitaminas y minerales. Las dos guías concuerdan en que se deben cubrir los requerimientos diarios y recomiendan evitar el uso de altas dosis de micronutrientes en ausencia de deficiencias específicas. La ESPEN recomienda que las necesidades energéticas se determinen mediante calorimetría indirecta, si no se dispone de este recurso, sugiere asumir que el gasto energético total de los pacientes con cáncer es similar al de sujetos sanos y generalmente oscila entre 25 y $30 \mathrm{kcal} / \mathrm{kg}$ / día. Recomienda además, la ingesta proteica superior a $1 \mathrm{~g} / \mathrm{kg} /$ día y, de ser posible hasta $1,5 \mathrm{~g} / \mathrm{kg} /$ día.

Las necesidades de micronutrientes deben determinarse de acuerdo con las recomendaciones y el cuadro clínico de cada paciente. Ejemplo de ello es el aumento en el requerimiento de zinc y vitamina $\mathrm{C}$ en el paciente quirúrgico, o bien el incremento en la necesidad de selenio en el paciente en estado crítico (Tabla 3 ).

\section{Intervenciones nutricionales generales y recomendaciones en cirugía, radioterapia, quimioterapia}

No hay evidencia acerca del momento óptimo para iniciar el soporte nutricional en el paciente con cáncer. Teniendo en cuenta que la desnutrición se asocia a un peor pronóstico y es difícil revertir la desnutrición en pacientes con cáncer con trastornos metabólicos, la terapia nutricional debe iniciarse, de preferencia, cuando los pacientes aún no están gravemente desnutridos y cuando los objetivos de la atención incluyen mantener o mejorar el estado nutricional. Se debe ofrecer apoyo nutricional a los pacientes que puedan desarrollar anorexia o defectos gastrointestinales debido a los efectos secundarios del tratamiento. A los pacientes con desnutrición severa que se someten a tratamiento activo se les debe ofrecer terapia nutricional de inmediato.

Las dos guías hacen énfasis en el enfoque integral que debe tener el asesoramiento nutricional; y por lo tanto, la intervención nutricional debe considerar y apuntar a mantener o mejorar los aspectos biológico, psicológico y social relacionados con la alimentación ya que esto tiene un impacto en la calidad de vida. Para ello, la ESPEN sugiere que se deben determinar hábitos y preferencias individuales. Además, un asesoramiento eficaz requiere habilidades de comunicación adecuadas para garantizar el alto cumplimiento de las recomendaciones nutricionales que se brindan en forma individual.

Las guías de la ESPEN y la Academia recomiendan la intervención nutricional que abarca desde los suple- 
Tabla 2. Recomendaciones sobre tamizaje, valoración nutricional, monitoreo y auditoria

\begin{tabular}{|c|c|}
\hline \multicolumn{2}{|c|}{ Tamizaje nutricional } \\
\hline Academia & ESPEN \\
\hline $\begin{array}{l}\text { Los pacientes deben someterse a tamizaje del estado nutricional } \\
\text { mediante una herramienta validada. } \\
\text { Grado de la recomendación: fuerte; imperativo. }\end{array}$ & $\begin{array}{l}\text { Para detectar alteraciones nutricionales en una etapa temprana } \\
\text { se recomienda evaluar regularmente la ingesta dietética y el } \\
\text { cambio de peso e IMC. } \\
\text { Nivel de evidencia: muy bajo. } \\
\text { Grado de la recomendación: fuerte. }\end{array}$ \\
\hline $\begin{array}{l}\text { Herramienta de tamizaje: } \\
\text { Se consideran válidas y confiables en pacientes hospitalizados: } \\
\text { MST, MSTC y MUST y en entornos ambulatorios / ambulatorios: } \\
\text { MST. } \\
\text { Grado de la recomendación: fuerte; imperativo. }\end{array}$ & $\begin{array}{l}\text { Herramienta de tamizaje: } \\
\text { No califica el grado de recomendación para alguna herramienta, } \\
\text { sin embargo, en los comentarios del apartado de tamizaje y } \\
\text { evaluación nutricional menciona como herramientas a } \\
\text { NRS-2002, MUST, MNA. }\end{array}$ \\
\hline \multicolumn{2}{|c|}{ Evaluación nutricional } \\
\hline $\begin{array}{l}\text { Utilizar una herramienta validada como parte de la evaluación } \\
\text { nutricional completa. } \\
\text { La Evaluación Global Subjetiva Generada por el Paciente } \\
\text { (PG-SGA) y Evaluación Global Subjetiva (SGA). } \\
\text { Grado de la recomendación: consenso; imperativo. }\end{array}$ & $\begin{array}{l}\text { En pacientes con tamizaje de riesgo, se recomienda evaluar de } \\
\text { forma objetiva y cuantitativa la ingesta nutricional, los síntomas } \\
\text { de impacto nutricional, masa muscular, el rendimiento físico y el } \\
\text { grado de inflamación sistémica. } \\
\text { Nivel de evidencia: muy bajo. } \\
\text { Grado de la recomendación: fuerte. }\end{array}$ \\
\hline $\begin{array}{l}\text { Dietética: } \\
\text { - Monitoreo dietético (tipo, cantidad y fuentes de alimentos). } \\
\text { - Patrón dietético. } \\
\text { - Intolerancias alimentarias. Consumo de medicamentos, } \\
\text { suplementos o tratamientos alternativos. } \\
\text { - Factores que afectan el acceso a alimentos. } \\
\quad \text { Grado de la recomendación: consenso; imperativo. }\end{array}$ & $\begin{array}{l}\text { Dietética: } \\
\text { - La reducción de la ingesta de alimentos se debe detectar de } \\
\text { forma temprana y analizada cualitativa y cuantitativamente. }\end{array}$ \\
\hline $\begin{array}{l}\text { Antropometría: } \\
\text { - Peso, talla, cambio de peso, IMC. } \\
\text { Grado de la recomendación: consenso; imperativo. }\end{array}$ & $\begin{array}{l}\text { Antropometría: } \\
\text { - El peso debe ser corregido en el caso de aquellos pacientes con } \\
\text { edema, ascitis o derrame pleural. } \\
\text { - La evaluación de las reservas musculares y de grasa pueden ser } \\
\text { obtenidas de diferentes vías, ejemplo: DEXA, antropometría, } \\
\text { tomografía computarizada a nivel lumbar } 3 \text { o bioimpedancia. }\end{array}$ \\
\hline $\begin{array}{l}\text { Bioquímicos: } \\
\text { - Datos bioquímicos. } \\
\text { - Pruebas de función Gl. } \\
\text { Grado de la recomendación: consenso; imperativo. }\end{array}$ & $\begin{array}{l}\text { Bioquímicos: } \\
\text { - La extensión de la inflamación sistémica se puede estimar } \\
\text { midiendo la proteína C reactiva en suero (PCR) y la albúmina. } \\
\text { La clasificación de la respuesta inflamatoria según el Glasgow } \\
\text { Prognostic Score modificado (mGPS) es altamente predictivo de } \\
\text { morbilidad y mortalidad en pacientes con cáncer. } \\
\text { No califica el grado de recomendación. }\end{array}$ \\
\hline $\begin{array}{l}\text { Exploración física: } \\
\text { Hallazgos físicos centrados en la nutrición como: } \\
\text { - Pérdida de masa muscular, pérdida de grasa subcutánea. } \\
\text { - Presencia de úlceras por presión o heridas. } \\
\text { - Síntomas de impacto nutricional como cambios en el apetito. } \\
\text { - Signos vitales. } \\
\text { - Cambio en los indicadores funcionales: escala de Karnofsky. } \\
\text { - Medición de fuerza y de agarre. } \\
\text { - Edema localizado o generalizado. } \\
\text { - Historia clínica personal y heredo familiar, incluyendo disfagia, } \\
\text { depresión y fatiga del dolor; tratamiento o terapia médica; } \\
\text { otras enfermedades, condiciones y enfermedades. } \\
\text { - Caquexia por cáncer. } \\
\text { - Historia social incluyendo factores psicológicos y } \\
\text { socioeconómicos. } \\
\text { Grado de la recomendación: consenso; imperativo. }\end{array}$ & $\begin{array}{l}\text { Exploración física: } \\
\text { - Examinar la presencia de síntomas con impacto en el consumo } \\
\text { de alimentos: xerostomía, cambios en el sentido del gusto } \\
\text { y olfato, náuseas, vómito, mucositis, constipación, diarrea, } \\
\text { trastorno de mala absorción, efectos secundarios de fármacos, } \\
\text { infecciones, dolor crónico o agudo, estrés psicológico, etc.). } \\
\text { - Se recomienda la utilización de la escala OMS/ECOG o } \\
\text { Karnofsky, además del uso de otras herramientas para } \\
\text { monitoreo diario de actividades o que evalúen de forma } \\
\text { cuantitativa el desarrollo físico o la función muscular. }\end{array}$ \\
\hline
\end{tabular}


Tabla 2. Recomendaciones sobre tamizaje, valoración nutricional, monitoreo y auditoria (Continuación)

\begin{tabular}{|c|c|}
\hline \multicolumn{2}{|c|}{ Monitoreo, auditoria y evaluación nutricional en pacientes adultos oncológicos } \\
\hline $\begin{array}{l}\text { Después de la intervención nutricional, para verificar el } \\
\text { progreso, un RDN debe monitorear y evaluar los siguientes } \\
\text { componentes de pacientes oncológicos adultos en cada visita } \\
\text { y comparar con los resultados individuales deseados relevantes } \\
\text { para el diagnóstico e intervención nutricional. Esto puede } \\
\text { incluir, pero no se limita a: } \\
\text { - Antropometría. } \\
\text { - Historia relacionada con la alimentación y la nutrición. } \\
\text { - Datos bioquímicos, pruebas médicas y procedimientos. } \\
\text { - Hallazgos físicos centrados en la nutrición. } \\
\text { - Historial del paciente. } \\
\text { - Historia social. } \\
\text { Grado de la recomendación: consenso; condicional. } \\
\text { Como parte de la monitoria y evaluación en pacientes con } \\
\text { cáncer de pulmón, páncreas, cabeza y cuello y Gl, o aquellos } \\
\text { con alto riesgo de pérdida de peso o pérdida involuntaria } \\
\text { de peso, un RDN debe monitorear y evaluar los síntomas de } \\
\text { impacto nutricional, marcadores de inflamación (valor elevado } \\
\text { de proteína C reactiva) y otros signos de desgaste que pueden } \\
\text { indicar precaquexia o caquexia por cáncer. }\end{array}$ & $\begin{array}{l}\text { Monitoreo de ingesta inadecuada, pérdida de peso y un bajo } \\
\text { IMC. En caso de encontrarse con riesgo, evaluar los síntomas de } \\
\text { impacto y trastornos metabólicos. } \\
\text { El monitoreo y la auditoría de la calidad del soporte nutricional y } \\
\text { metabólico están en sus inicios. Algunos criterios de auditoria: } \\
\text { - El porcentaje de pacientes a quien se realiza tamizaje del riesgo } \\
\text { de desnutrición debe superar por ejemplo, } 80 \% \text {. } \\
\text { - El porcentaje de pacientes con cáncer con un resultado de } \\
\text { tamizaje de alto riesgo que reciben evaluación nutricional } \\
\text { adicional debe superar por ejemplo, } 80 \% \text {. } \\
\text { - El porcentaje de pacientes con cáncer sometidos a una } \\
\text { evaluación nutricional en la que se estima la masa muscular } \\
\text { debería superar por ejemplo, } 80 \% \text {. } \\
\text { - El porcentaje de pacientes con cáncer con un resultado de } \\
\text { tamizaje de alto riesgo que reciben terapia nutricional para } \\
\text { mejorar la ingesta de energía y proteínas debe superar por } \\
\text { ejemplo, } 80 \% \text {. } \\
\text { Nivel de evidencia: bajo. } \\
\text { Grado de la recomendación: fuerte. }\end{array}$ \\
\hline & \\
\hline
\end{tabular}

Tabla 3. Recomendaciones de requerimiento energético y substratos

\begin{tabular}{|c|c|c|}
\hline \multicolumn{3}{|c|}{ Academia } \\
\hline Vitaminas & \multicolumn{2}{|c|}{$\begin{array}{l}\text { Cubrir la demanda diaria, aunque se desconoce el requerimiento diario de micronutrientes para un paciente } \\
\text { con cáncer }\end{array}$} \\
\hline \multicolumn{3}{|c|}{ ESPEN } \\
\hline & Recomendación & $\begin{array}{l}\text { Nivel de evidencia } \\
\text { y fuerza de la } \\
\text { recomendación }\end{array}$ \\
\hline $\begin{array}{l}\text { Gasto energético } \\
\text { total }\end{array}$ & $\begin{array}{l}\text { Si no se mide en forma individual, se podría asimilar a sujetos sanos y } \\
\text { generalmente oscila entre } 25 \text { y } 30 \mathrm{kcal} / \mathrm{kg} / \mathrm{d} \text { día. }\end{array}$ & $\begin{array}{l}\text { Nivel: bajo } \\
\text { Recomendación: fuerte }\end{array}$ \\
\hline $\begin{array}{l}\text { Requerimiento de } \\
\text { proteína }\end{array}$ & $\begin{array}{l}\text { Se recomienda ingesta por encima de } 1 \mathrm{~g} / \mathrm{kg} / \text { día y, si es posible hasta } 1,5 \mathrm{~g} / \mathrm{kg} / \mathrm{día} \text {. } \\
\text { En sujetos con función renal normal, la ingesta de proteínas en dosis de hasta, y } \\
\text { por encima, de } 2 \mathrm{~g} / \mathrm{kg} / \text { día es segura. } \\
\text { En pacientes con insuficiencia renal aguda o crónica, el aporte proteico no debe } \\
\text { exceder } 1,0 \text { o } 1,2 \mathrm{~g} / \mathrm{kg} / \text { día, respectivamente. }\end{array}$ & $\begin{array}{l}\text { Nivel: moderado } \\
\text { Recomendación: fuerte }\end{array}$ \\
\hline $\begin{array}{l}\text { Elección de } \\
\text { sustratos } \\
\text { energéticos }\end{array}$ & $\begin{array}{l}\text { La proporción óptima de carbohidratos y grasa en pacientes con cáncer no se ha } \\
\text { determinado, pero puede derivarse de argumentos fisiopatológicos. } \\
\text { Pacientes con resistencia a la insulina que presentan pérdida de peso, se } \\
\text { recomienda aumentar la densidad energética de la dieta y reducir la carga } \\
\text { glicémica aumentando el aporte de grasas en lugar de hidratos de carbono. }\end{array}$ & $\begin{array}{l}\text { Nivel: bajo } \\
\text { Recomendación: fuerte }\end{array}$ \\
\hline $\begin{array}{l}\text { Vitaminas y } \\
\text { minerales }\end{array}$ & $\begin{array}{l}\text { Se recomienda que sean suministradas en cantidades aproximadamente iguales } \\
\text { a la RDA y aconseja evitar el uso de altas dosis de micronutrientes en ausencia } \\
\text { de deficiencias específicas. }\end{array}$ & $\begin{array}{l}\text { Nivel: bajo } \\
\text { Recomendación: fuerte }\end{array}$ \\
\hline
\end{tabular}


mentos nutricionales orales hasta la utilización de la vía enteral y parenteral si fuese necesario. Además, la ESPEN brinda recomendaciones para pacientes que reciben tratamiento quirúrgico, acerca de las dietas potencialmente dañinas, el síndrome de realimentación y la nutrición a domicilio. Las recomendaciones de las dos guías tienen en cuenta el paciente que recibe quimio y radioterapia. La Academia preconiza intervenciones nutricionales en caso de trasplante de médula ósea, de neuropatía y neutropenia (Tablas 4 y 5 ).

Las guías de la ESPEN recomiendan para el paciente con tratamiento quirúrgico realizar el cuidado nutricional dentro del protocolo ERAS que se ha desarrollado desde hace una década y que ha mostrado tener un buen nivel de evidencia.

\section{Recomendaciones de actividad física y farmacológicas}

Las guías de la ESPEN abarcan el enfoque multimodal de las intervenciones en el paciente con cáncer y proporciona recomendaciones para mejorar y aumentar la actividad física de los pacientes y modular la ingesta de alimentos, la respuesta inflamatoria y metabólica con estrategias farmacológicas. Es importante mencionar que el papel de la actividad física en estos pacientes cada vez se hace más relevante. Los ensayos aleatorios publicados y resumidos en varios meta-análisis proporcionan evidencia relativamente sólida de que la actividad física es bien tolerada y segura en las diferentes etapas del cáncer.

Tabla 4. Intervenciones nutricionales

\begin{tabular}{|c|c|}
\hline \multicolumn{2}{|r|}{ Academia } \\
\hline $\begin{array}{l}\text { Eficacia de la } \\
\text { intervención } \\
\text { nutricional }\end{array}$ & $\begin{array}{l}\text { En pacientes oncológicos adultos que han sido identificados con precaquexia o caquexia por cáncer, una } \\
\text { RDN debe iniciar una intervención pronta y agresiva para abordar los síntomas de la nutrición y preservar o } \\
\text { prevenir la pérdida de peso y masa magra. } \\
\text { Grado de la recomendación débil; condicional. }\end{array}$ \\
\hline \multicolumn{2}{|r|}{ ESPEN } \\
\hline $\begin{array}{l}\text { Eficacia de la } \\
\text { intervención } \\
\text { nutricional }\end{array}$ & $\begin{array}{l}\text { Se recomienda la intervención nutricional para aumentar la ingesta oral en pacientes con cáncer que } \\
\text { pueden comer pero están desnutridos o en riesgo de desnutrición. Esto incluye asesoramiento dietético, } \\
\text { el tratamiento de los síntomas y trastornos que afectan la ingesta de alimentos y los suplementos } \\
\text { nutricionales orales. } \\
\text { Nivel de evidencia: moderado } \\
\text { Grado de la recomendación: fuerte. }\end{array}$ \\
\hline $\begin{array}{l}\text { Dietas } \\
\text { potencialmente } \\
\text { dañinas }\end{array}$ & $\begin{array}{l}\text { Se recomienda no utilizar estrategias dietéticas que restrinjan la ingesta de energía en pacientes con o en } \\
\text { riesgo de desnutrición. } \\
\text { Nivel de evidencia: bajo } \\
\text { Grado de la recomendación: fuerte. }\end{array}$ \\
\hline $\begin{array}{l}\text { Vías de alimentación: } \\
\text { cuándo escalar }\end{array}$ & $\begin{array}{l}\text { Si se ha tomado una decisión para alimentar a un paciente, se recomienda la nutrición enteral si la nutrición } \\
\text { oral sigue siendo inadecuada a pesar de las intervenciones nutricionales (asesoramiento, suplementos), y la } \\
\text { nutrición parenteral si la nutrición enteral no es suficiente o factible. } \\
\text { Nivel de evidencia: moderado } \\
\text { Grado de la recomendación: fuerte. }\end{array}$ \\
\hline $\begin{array}{l}\text { Nutrición artificial a } \\
\text { domicilio }\end{array}$ & $\begin{array}{l}\text { En pacientes con ingesta dietética crónica insuficiente y malabsorción incontrolable, } \\
\text { recomiendan nutrición artificial domiciliaria (ya sea enteral o parenteral) en pacientes indicados. } \\
\text { Nivel de evidencia: bajo } \\
\text { Grado de la recomendación: fuerte. }\end{array}$ \\
\hline $\begin{array}{l}\text { Sindrome de } \\
\text { realimentación }\end{array}$ & $\begin{array}{l}\text { Si la ingesta oral de alimentos ha disminuido severamente durante un período prolongado se recomienda } \\
\text { aumentar la nutrición (oral, enteral o parenteral) en forma lenta durante varios días y tomar precauciones } \\
\text { adicionales para prevenir un síndrome de realimentación. } \\
\text { Nivel de evidencia: bajo } \\
\text { Grado de la recomendación: fuerte. }\end{array}$ \\
\hline
\end{tabular}


Tabla 5. Recomendaciones sobre terapia nutricional en radioterapia, quimioterapia y cirugía

\begin{tabular}{|c|c|}
\hline \multicolumn{2}{|c|}{ Radioterapia } \\
\hline Academia & ESPEN \\
\hline $\begin{array}{l}\text { La terapia médico nutricional ha demostrado ser eficaz y brindar } \\
\text { múltiples beneficios en pacientes sometidos a quimio (QT) o } \\
\text { radioterapia (RT) en pacientes ambulatorios u hospitalizados. } \\
\text { Grado de la recomendación: fuerte; condicional. }\end{array}$ & Información no disponible en la guía de la ESPEN. \\
\hline \multicolumn{2}{|c|}{ Quimioterapia } \\
\hline Academia & ESPEN \\
\hline $\begin{array}{l}\text { Los expertos en nutrición deben ser miembros de equipos } \\
\text { interdisciplinarios, proporcionar terapia multimodal a } \\
\text { pacientes adultos de oncología sometidos a QT o RT. } \\
\text { Grado de la recomendación: razonable; condicional. } \\
\text { Se debería aconsejar al paciente el uso de vitamina E, calcio } \\
\text { y magnesio acetil-L-carnitina, GLN y glutatión, estos pueden } \\
\text { o no ser beneficiosos como medio de prevenir o mejorar la } \\
\text { neuropatía periférica inducida por quimioterapia. } \\
\text { Grado de la recomendación: débil; condicional. } \\
\text { Precauciones dietéticas en pacientes con neutropenia: se debe } \\
\text { proporcionar asesoramiento dietético sobre la manipulación } \\
\text { higiénica de alimentos y cuáles de ellos puede presentar riesgos } \\
\text { infecciosos durante el período de neutropenia. } \\
\text { Una dieta neutropénica no es necesaria, pero la consejería } \\
\text { de alimentos seguros se recomienda como una medida de } \\
\text { precaución. } \\
\text { Grado de la recomendación: razonable; condicional. } \\
\text { Si un paciente de oncología adulto es sometido a trasplante de } \\
\text { médula ósea, se debe proporcionar asesoría dietética sobre la } \\
\text { seguridad de la manipulación de alimentos y sobre alimentos } \\
\text { que pueden presentar riesgos infecciosos durante el período de } \\
\text { neutropenia. } \\
\text { Grado de la recomendación: débil; condicional. }\end{array}$ & $\begin{array}{l}\text { Durante el tratamiento farmacológico contra el cáncer se } \\
\text { recomendaron asegurar una ingesta nutricional adecuada y } \\
\text { mantener la actividad física. } \\
\text { Nivel de evidencia: muy bajo. } \\
\text { Grado de la recomendacion: fuerte. } \\
\text { En un paciente sometido a tratamiento farmacológico curativo } \\
\text { contra el cáncer, si la ingesta oral de alimentos es inadecuada a } \\
\text { pesar del asesoramiento y los suplementos nutricionales orales, } \\
\text { recomendamos la nutrición enteral suplementaria o, si esto no } \\
\text { es suficiente o posible, parenteral. } \\
\text { Nivel de evidencia: muy bajo. } \\
\text { Grado de la recomendacion: fuerte. } \\
\text { Durante la quimioterapia intensiva y después del trasplante } \\
\text { de células madre, recomendamos mantener la actividad física } \\
\text { y garantizar una ingesta nutricional adecuada. Esto puede } \\
\text { requerir nutrición enteral, parenteral o mixta. } \\
\text { Nivel de evidencia: muy bajo. } \\
\text { Grado de la recomendacion: fuerte. } \\
\text { No hay datos clínicos consistentes suficientes para recomendar } \\
\text { una dieta bacteriana baja (para pacientes neutropénicos) } \\
\text { para los pacientes con más de } 30 \text { días después del trasplante } \\
\text { alogénico. } \\
\text { Nivel de evidencia: bajo. } \\
\text { Grado de la recomendación: - }\end{array}$ \\
\hline \multicolumn{2}{|c|}{ ESPEN Cirugía } \\
\hline \multicolumn{2}{|c|}{$\begin{array}{l}\text { Para todos los pacientes con cáncer sometidos a cirugía curativa o paliativa, se recomienda la administración de cuidado } \\
\text { nutricional dentro de un programa de recuperación mejorada después de la cirugía (ERAS); dentro de este programa, cada } \\
\text { paciente debe someterse a exámenes de detección de desnutrición y, si se lo considera en riesgo, debe recibir apoyo nutricional } \\
\text { adicional. } \\
\text { Nivel de evidencia: alto. } \\
\text { Grado de la recomendación: fuerte. } \\
\text { Para pacientes que son reintervenidos, se recomienda el manejo de cada episodio quirúrgico dentro de un programa ERAS. } \\
\text { Nivel de evidencia: bajo. } \\
\text { Grado de la recomendación: fuerte. } \\
\text { En pacientes con cáncer quirúrgico con riesgo de desnutrición o que ya están desnutridos, se recomienda el apoyo nutricional } \\
\text { adecuado tanto durante la atención hospitalaria como después del alta hospitalaria. } \\
\text { Nivel de evidencia: moderado. } \\
\text { Grado de la recomendación: fuerte. }\end{array}$} \\
\hline
\end{tabular}


Las estrategias farmacológicas se limitan al control de síntomas como el apetito y aunque en las últimas décadas se ha investigado acerca de posibles dianas terapéuticas para disminuir el desgaste muscular característico de la caquexia, hoy en día ninguna estrategia farmacológica está disponible.

\section{Recomendaciones de fármaco-nutrimentos}

Uno de los temas de mayor controversia desde hace algunas décadas es el uso de terapia nutricional enri- quecida con inmuno-nutrimentos. Las dos guías recomiendan el uso de aceite de pescado, pero con nivel de evidencia diferente. La Academia afirma que el nivel de evidencia científica es fuerte, mientras que la ESPEN la clasifica como débil (Tabla 6). El aceite de pescado (dosis más frecuentemente utilizadas: $4 \mathrm{~g}-6 \mathrm{~g} / \mathrm{dí}$ ) y los ácidos grasos omega-3 de cadena larga (1 g - 2 g/ día) disminuyen la respuesta inflamatoria en pacientes con cáncer como lo demuestra el descenso en los marcadores inflamatorios (interleucina 6 o proteína $\mathrm{C}$ reactiva) y el gasto energético en reposo.

Tabla 6. Recomendaciones de farmaconutrientes

\begin{tabular}{|c|c|c|}
\hline & Academia & ESPEN \\
\hline $\begin{array}{l}\text { Aceite de pescado y } \\
\text { pérdida de peso }\end{array}$ & $\begin{array}{l}\text { Si en el paciente oncológico adulto existen } \\
\text { síntomas y la ingesta dietética es inadecuada o } \\
\text { subóptima, además de experimentar pérdida } \\
\text { de peso y masa libre de grasa, puede considerar } \\
\text { el uso de suplementos y de alimentos con } \\
\text { propósitos médicos especiales que contengan } \\
\text { EPA como un componente de la intervención } \\
\text { nutricional. } \\
\text { Grado de la recomendación: fuerte; imperativo. }\end{array}$ & $\begin{array}{l}\text { En pacientes con cáncer avanzado sometidos a } \\
\text { quimioterapia y riesgo de pérdida de peso o desnutridos, } \\
\text { se sugiere utilizar suplementos con cadena larga de } \\
\text { ácidos grasos N-3 o aceite de pescado para estabilizar } \\
\text { o mejorar el apetito, la ingesta de alimentos, la masa } \\
\text { corporal magra y el peso corporal. } \\
\text { Nivel de evidencia: bajo } \\
\text { Grado de la recomendación: baja. }\end{array}$ \\
\hline $\begin{array}{l}\text { Inmunonutrición } \\
\text { (arginina, ácidos } \\
\text { grasos N-3, } \\
\text { nucleótidos) }\end{array}$ & $\begin{array}{l}\text { Información no disponible en la guía de la } \\
\text { Academia. }\end{array}$ & $\begin{array}{l}\text { En pacientes con cáncer Gl superior sometidos a } \\
\text { resección quirúrgica en el contexto de la atención } \\
\text { perioperatoria tradicional, se recomienda la } \\
\text { inmunonutrición oral / enteral. } \\
\text { Nivel de evidencia: alto } \\
\text { Grado de la recomendación: fuerte. }\end{array}$ \\
\hline $\begin{array}{l}\text { Glutamina }(G L N) \\
\text { parenteral y oral }\end{array}$ & $\begin{array}{l}\text { Se recomienda el uso de GLN parenteral para } \\
\text { prevenir o tratar la mucositis oral en pacientes } \\
\text { oncológicos con tumores sólidos, los RDN deben } \\
\text { informar que su uso podría ser beneficioso o no. } \\
\text { Efectividad no establecida. } \\
\text { En pacientes con trasplante de células madre un } \\
\text { RDN podría recomendar o no, GLN parenteral en } \\
\text { dosis que van desde } 0,2-0,5 \mathrm{~g} / \mathrm{kg} / \text { día. } \\
\text { Grado de la recomendación: razonable; } \\
\text { condicional. }\end{array}$ & $\begin{array}{l}\text { No hay datos clínicos consistentes suficientes para } \\
\text { recomendar suplementación de glutamina } \\
\text { - Durante la terapia convencional citotóxica o dirigida. } \\
\text { - Para mejorar resultado clínico en pacientes sometidos } \\
\text { a altas dosis de quimioterapia y trasplante de células } \\
\text { madre hematopoyéticas. } \\
\text { - Para prevenir la enteritis o la diarrea inducida por la } \\
\text { radiación, la estomatitis, la esofagitis o la toxicidad de } \\
\text { la piel. } \\
\text { Nivel de evidencia: bajo } \\
\text { Grado de la recomendación: no específica. }\end{array}$ \\
\hline $\begin{array}{l}\text { Aminoácidos de } \\
\text { cadena ramificada }\end{array}$ & $\begin{array}{l}\text { Información no disponible en la guía de la } \\
\text { Academia. }\end{array}$ & $\begin{array}{l}\text { No hay datos clínicos consistentes suficientes para } \\
\text { recomendar la suplementación con aminoácidos de } \\
\text { cadena ramificada u otros aminoácidos o metabolitos } \\
\text { (HMB) para mejorar la masa libre de grasa. } \\
\text { Nivel de evidencia: bajo } \\
\text { Grado de la recomendación: no específica. }\end{array}$ \\
\hline Probióticos & $\begin{array}{l}\text { Información no disponible en la guía de la } \\
\text { Academia. }\end{array}$ & $\begin{array}{l}\text { No hay datos clínicos consistentes suficientes para } \\
\text { recomendar el uso de probióticos para reducir la diarrea } \\
\text { inducida por la radiación. } \\
\text { Nivel de evidencia: bajo } \\
\text { Grado de la recomendación: no específica. }\end{array}$ \\
\hline
\end{tabular}


Existe una diferencia importante en la recomendación de uso de glutamina en las dos guías analizadas. La Academia considera que la glutamina parenteral en dosis farmacológicas puede ser beneficiosa en pacientes sometidos a trasplante de células hematopoyéticas y recomienda dosis que van de $0,2 \mathrm{~g}$ a $0,5 \mathrm{~g} / \mathrm{kg} /$ día por vía intravenosa. Según la Academia, la evidencia indica que la glutamina parenteral debe iniciarse temprano en el curso del tratamiento. La glutamina parenteral se asocia a un mejor balance de nitrógeno y a una disminución de la morbilidad. Sin embargo, la disminución en la estancia hospitalaria se encontró solo cuando los datos de alo-trasplante y trasplantes autólogos se combinaron. Para ESPEN, no hay evidencia suficiente para recomendar su utilización. La guía de ESPEN afirma que teniendo en cuenta que la glutamina participa en diversas vías metabólicas y no hay suficiente evidencia científica concluyente, es prudente evitar su administración a largo plazo en la caquexia del cáncer.

\section{Cuidado nutricional en el paciente sobreviviente y en cuidados paliativos}

Las guías de la ESPEN recomiendan mantener un peso saludable (IMC $18,5-25 \mathrm{~kg} / \mathrm{m}^{2}$ ) en los sobrevivientes de cáncer; así como mantener un estilo de vida saludable, que incluye estar físicamente activo y una dieta basada en vegetales, frutas y granos integrales, $y$ baja en grasas saturadas, carne roja y alcohol (nivel de evidencia bajo y grado de recomendación fuerte). En los pacientes en estado avanzado se recomienda evaluar de forma rutinaria e identificar a todos los pacientes con ingesta nutricional inadecuada, pérdida de peso e IMC bajo, y si se encuentran en riesgo, realizar una evaluación completa con el fin de detectar síntomas de impacto nutricional tratables y trastornos metabólicos (nivel de evidencia bajo y grado de recomendación fuerte). En estos pacientes se recomienda ofrecer e implementar intervenciones nutricionales solo después de considerar, junto con el paciente, el pronóstico de la enfermedad maligna y el beneficio esperado en la calidad de vida y potencial supervivencia, así como la carga asociada a la atención nutricional (nivel de evidencia bajo y grado de recomendación fuerte).

En los pacientes en fase terminal se recomienda basar su tratamiento en el confort. Es poco probable que la hidratación artificial y la nutrición proporcionen algún beneficio para la mayoría de los pacientes. Sin embargo, en estados de confusión agudos, sugieren utilizar una hidratación corta y limitada para descartar la deshidratación como causa precipitante (nivel de evidencia bajo y grado de recomendación fuerte).

Los aspectos bioéticos de la nutrición en pacientes en estado terminal son abordados por las guías de la ESPEN: para los pacientes con enfermedad avanzada que se espera que sobrevivan semanas o días se debe considerar a la persona enferma como un ser integral con un acervo religioso, cultural y étnico individual y por otro, los aspectos sociales, emocionales y existenciales relacionados con la alimentación. La decisión de no iniciar alimentación artificial en un paciente que no puede consumir alimentos, en general, se considera solo al final de la vida. Hay datos que muestran los beneficios de la nutrición artificial domiciliaria en pacientes con cáncer con alteración crónica de la ingesta o absorción de la dieta, incluso en cáncer avanzado, siempre que haya una supervivencia de más de unas pocas semanas. El beneficio puede deducirse con claridad del hecho de que algunos pacientes con cáncer sobreviven varios meses e incluso años exclusivamente con nutrición parenteral, es decir, períodos de tiempo durante los cuales cualquier persona sin alimentos habría sucumbido a la inanición.

\section{CONCLUSIÓN}

Las guías de práctica clínica pretenden ofrecer una forma de cerrar la brecha entre las políticas, las mejores prácticas, los contextos locales y las decisiones del paciente. Los programas que buscan mejorar la calidad del cuidado nutricional en pacientes con cáncer deben estar vinculados con las guías ya que las recomendaciones basadas en la evidencia constituyen la base para identificar las prácticas con las cuales se pueden obtener los mejores resultados. La mayoría de las recomendaciones de la Academia y de la ESPEN se basan en consensos, tienen un nivel de evidencia bajo o muy bajo lo que muestra que este campo aún está en pleno desarrollo. Sin embargo, aunque presentan diferencias en ciertos temas, son una herramienta útil que debe orientar la práctica del cuidado nutricional en oncología.

\section{Financiación}

El presente estudio no tuvo financiación.

\section{Conflicto de intereses}

Los autores declaran no tener conflicto de intereses. 


\section{Referencias bibliográficas}

1. Organización Mundial de la Salud. 70.a ASAMBLEA MUNDIAL DE LA SALUD WHA70.12. Prevención y control del cáncer en el contexto de un enfoque integrado. 31 de mayo de 2017. [Internet]. [Consultado el 28 de marzo de 2018]. Disponible en:http://apps.who.int/gb/ebwha/ pdf_files/WHA70/A70_R12-sp.pdf.

2. Hébuterne X, Lemarié E, Michallet M, de Montreuil CB, Schneider SM, Goldwasser F. Prevalence of malnutrition and current use of nutrition support in patients with cancer. JPEN J Parenter Enteral Nutr. 2014; 38(2):196-204.

3. Planas M, Álvarez-Hernández J, León-Sanz M, Celaya-Pérez S, Araujo K, García de Lorenzo A, et al. Prevalence of hospital malnutrition in cancer patients: a sub-analysis of the PREDyCES ${ }^{\circledast}$ study. Support Care Cancer. 2016; 24(1): 429-35.

4. Baracos V, Kazemi-Bajestani SM. Clinical outcomes related to muscle mass in humans with cancer and catabolic illnesses. Int J Biochem Cell Biol. 2013;45(10): 2302-8.

5. Martin L, Birdsell L, Macdonald N, Reiman T, Clandinin MT, McCargar LJ, et al. Cancer cachexia in the age of obesity: skeletal muscle depletion is a powerful prognostic factor, independent of body mass index. J Clin Oncol. 2013;31(12):1539-47.

6. Martin L, Senesse P, Gioulbasanis I, Antoun S, Bozzetti F, Deans C, et al. Diagnostic criteria for the classification of cancer-associated weight loss. J Clin Oncol. 2015;33(1):90-9.

7. Attar A, Malka D, Sabaté JM, Bonnetain F, Lecomte T, Aparicio T, et al. Malnutrition is high and underestimated during chemotherapy in gastrointestinal cancer: an AGEO prospective cross-sectional multicenter study. Nutr Cancer. 2012;64(4):535-42.

8. Gyan E, Raynard B, Durand JP, Lacau Saint Guily J, Gouy S, Movschin ML, et al. Malnutrition in patients with cancer. JPEN J Parenter Enteral Nutr. 2017; 1: 1:148607116688881.

9. Preiser JC, Schneider SM. ESPEN disease-specific guideline framework. Clin Nutr. 2011; 30(5):549-52.

10. Arends J, Bachmann P, Baracos V, Barthelemy N, Bertz H, Bozzetti $\mathrm{F}$, et al. ESPEN guidelines on nutrition in cancer patients. Clin Nutr. 2017; 36(1):11-48.

11. Arends J, Bodoky G, Bozzetti F, Fearon K, Muscaritoli M, Selga $G$, et al. ESPEN guidelines on enteral nutrition: nonsurgical oncology. Clin Nutr. 2006;25(2):245-59.

12. August DA, Huhmann MB, American Society for Parenteral and Enteral Nutrition (A.S.P.E.N.) Board of Directors. A.S.P.E.N. clinical guidelines: nutrition support therapy during adult anticancer treatment and in hematopoietic cell transplantation. JPEN J Parenter Enter Nutr. 2009;33(5):472-500.

13. Bozzetti F, Arends J, Lundholm K, Micklewright A, Zurcher G, Muscaritoli M; ESPEN. ESPEN guidelines on parenteral nutrition: non-surgical oncology. Clin Nutr. 2009; 28(4):445-54.
14. Kushi LH, Doyle C, McCullough M, Rock CL, DemarkWahnefried W, Bandera EV, et al. American cancer society guidelines on nutrition and physical activity for cancer prevention: reducing the risk of cancer with healthy food choices and physical activity. CA Cancer J Clin. 2012; 62(1):30-67.

15. Thompson KL, Elliott L, Fuchs-Tarlovsky V, Levin RM, Voss AC, Piemonte T. Oncology Evidence-Based Nutrition Practice Guideline for Adults. J Acad Nutr Diet. 2017; 117(2):297-310.

16. Institute of Medicine of the National Academies. Clinical Practice Guidelines We Can Trust. 2011. Washington, DC: The National Academies Press. [Internet]. [Consultado el 1 de agosto de 2018]. Disponible en : http://data.care-statement.org/wp-content/ uploads/2016/12/IOMGuidelines-2013-1.pdf.

17. Brozek JL, Akl EA, Alonso-Coello P, Lang D, Jaeschke R, Williams JW, et al. Grading quality of evidence and strength of recommendations in clinical practice guidelines. Part 1 of 3. An overview of the GRADE approach and grading quality of evidence about interventions. Allergy. 2009;64(5):669-77.

18. Handu D, Moloney L, Wolfram T, Ziegler P, Acosta A, Steiber A. A. Academy of Nutrition and Dietetics Methodology for Conducting Systematic Reviews for the Evidence Analysis Library. J Acad Nutr Diet. 2016;116(2):311-8.

19. Academy of Nutrition and Dietetics. Evidence Analysis Library. Oncology guideline 2013. [Internet]. [Consultado el 19 de abril de 2018]. Disponible en: https://www.andeal.org/ topic.cfm?menu=5291\&amp;cat=5066.

20. Kredo T, Bernhardssong S, Machingaidze SH, Young T, Louw Q, Ochodo E, et al. Guide to clinical practice guidelines: the current state of play. Int J Qual Health Care. 2016; 28(1):122-8.

21. Agency for Healthcare Research and Quality. Oncology evidence-based nutrition practice guideline. [Internet]. [Consultado el 19 de abril de 2018]. Disponible en: https:// www.guideline.gov/summaries/summary/48765/oncologyevidencebased-nutrition-practice-guideline? $\mathrm{q}=$ Nutrition.

22. Academy of Nutrition and Dietetics. What is a Registered Dietitian Nutritionist. [Internet]. [Consultado el 30 de marzo de 2018]. Disponible en: https://www.eatrightpro. org/about-us/what-is-an-rdn-and-dtr/what-is-a-registereddietitian-nutritionist.

23. Hammond MI, Myers EF, Trostler N. Nutrition care process and model: an academic and practice odyssey. J Acad Nutr and Diet. 2014; 114(12):1879-94.

24. Cederholm T, Barazzoni R, Austin P, Ballmer P, Biolo G, Bischoff SC, et al. ESPEN guidelines on definitions and terminology of clinical nutrition. Clin Nutr. 2017;36(1):49-64. 